\title{
Experiencia del profesional de enfermería ante la muerte y el proceso de morir en unidades de cuidado intensivo
}

\author{
Valle-Figueroa, María del Carmen ${ }^{\text {; }}$; García-Puga, Julio Alfredo; Quintana-Zavala, María Olga; \\ García-Pérez, Yosselyn ${ }^{4}$
}

\begin{abstract}
RESUMEN
El objetivo de la presente investigación es comprender la experiencia del profesional de enfermería ante el proceso de muerte. Metodología: Estudio cualitativo, descriptivo y transversal. Los participantes fueron 7 enfermeras de unidad de cuidados intensivos de una institución de segundo nivel. Para seleccionarlos se construyó una clasificación tipológica con: edad del paciente, tiempo de relación, experiencia ante procesos de muerte y edad de la enfermera. El análisis de datos se llevó a cabo de forma inductiva y deductiva, se utilizó el programa NVivo versión 7. La presente investigación se apegó a lo establecido en el Reglamento de la Ley General de Salud en Materia de Investigación. Resultados: Las participantes informan que las principales estrategias de afrontamiento ante la muerte y proceso de morir son alejamiento o distanciamiento emocional, así como búsqueda de apoyo social en grupos religiosos, ayuda psicológica o grupos de tanatología. Los mecanismos innatos se constituyen en los capitales culturales mientras los adquiridos suelen ser incorporados a partir de las instituciones educativas y de salud. Conclusiones: El proceso de morir se determina por tres tipos de estímulos contextuales importantes: edad del paciente, relación enfermera paciente y tiempo de ésta. Se encontraron procesos de afrontamiento eficaces en aquellas enfermeras que dentro de su preparación profesional han incluido capacitación sobre tanatología. Las principales estrategias de afrontamiento encontrados fueron: redefinición acerca de la muerte y soporte emocional por algún tipo de creencia religiosa.
\end{abstract}

Palabras clave: Muerte; atención de enfermería; personal de enfermería (DeCS).

'Universidad de Sonora, División de Ciencias Biológicas y de la Salud, Departamento de Enfermería, Orcid: 0000-0002-8154-2818. E-mail: maria.valle@unison.mx 2Universidad de Sonora, División de Ciencias Biológicas y de la Salud, Departamento de Enfermería, Orcid: 0000-0001-5207-7454. 3universidad de Sonora, División de Ciencias Biológicas y de la Salud, Departamento de Enfermería, Orcid: 0000-0003-3725-4226 ${ }^{4}$ Hospital General de Guaymas.

Recibido: 27/01/2019

Aceptado: 27/03/2019

*Autor para correspondencia

\section{Cómo citar este artículo}

Valle-Figueroa MC, García-Puga JA, Quintana-Zavala MO, García-Pérez Y. Experiencia del profesional de enfermería ante la muerte y el proceso de morir en unidades de cuidado intensivo. SANUS. 2019; (11): 19-31. [Acceso_____ ]; Disponible en: 


\title{
Experiência do profissional de enfermagem em matéria de mor- te e processo de morrer em unidades de atenção intensivas
}

\begin{abstract}
RESUMO
Objetivo: $\bigcirc$ objetivo desta pesquisa é compreender a experiência do profissional de enfermagem em relação à morte de um paciente. Metodologia: Isso foi um estudo qualitativo, descritivo e transversal. Participaram sete enfermeiras da unidade de terapia intensiva de uma instituição de segundo nível. Para selecionar os participantes, uma classificação tipológica foi construída incluindo idade do paciente, duração do relacionamento, experiência em processos de morte e idade da enfermeira. A análise dos dados foi realizada de maneira indutiva-dedutiva; foi utilizado o software Nvivo, versão 7. Esta pesquisa está alinhada com o Regulamento da Lei Geral de Saúde na Área de Pesquisa em Saúde. Resultados: Os participantes informaram que as principais estratégias de enfrentamento em relação à morte e ao processo de morrer são distanciamento ou afastamento emocional, bem como buscar apoio social em grupos religiosos, ajuda psicológica ou grupos de tanatologia. Mecanismos inatos fazem parte das capitais culturais, enquanto os adquiridos são geralmente ensinados em instituições de educação e saúde. Conclusões: $\bigcirc$ processo de morrer é determinado por três tipos de estímulos contextuais importantes: Idade do paciente, relação enfermeira-paciente e duração desse relacionamento. Processos de enfrentamento eficientes foram encontrados naquelas enfermeiras que, em sua preparação profissional, tanatologia foi incluída. As principais estratégias de enfrentamento encontradas foram: redefinição da morte e apoio emocional através de algum tipo de crença religiosa.
\end{abstract}

Palavras chave: Morte; cuidados de enfermagem; pessoal de enfermagem (DeCS).

\section{INTRODUCCIÓN}

En el ejercicio diario del profesional de la salud, especialmente en cuidados intensivos, existe un factor que es profundamente estresante: vivir en un constante desafío entre la vida y la muerte; con consecuencias de desgaste emocional y físico(1). La muerte como proceso natural es parte de la vida y solo en el año 2018, se registraron 14,916 defunciones en el ámbito hospitalario en los 72 municipios del Estado de Sonora, de las cuales 8,747 fueron personas de sexo masculino y 6,157 fueron de sexo femenino ${ }^{(2)}$. De las principales causas de muerte se encuentran los infartos al miocardio, enfermedades crónico-degenerativas, como la Diabetes Mellitus y los accidentes automovilísticos. Con independencia de la causa, cualquier muerte es traumática para las familias y el profesional de salud, ya que suelen ser repentinas o conllevan un largo proceso de sufrimiento.

Las unidades de cuidados intensivos constituyen áreas donde los pacientes cursan con una larga estadía y ocurre un número significativo de defunciones, por lo cual el profesional de enfermería se ve obligado a utilizar las herramientas que recibieron durante su preparación académica para afrontar dichas experiencias ${ }^{(3,-5)}$. Desde un punto de vista social, la muerte es un tema hasta cierto punto velado en las discusiones cotidianas de las personas, ello, en parte porque recuerda su finitud y la incertidumbre ante este proceso, lo que puede condicionar angustia existencial. Dicho proceso de muerte es una de las situaciones de mayor impacto en el personal sanitario y en especial de Enfermería, quien debe aplicar diversos mecanismos de afrontamiento ante tal evento(1).

Para comprender este fenómeno de adaptación al fenómeno de muerte, la presente investigación utilizó el modelo de adaptación de Sor Callista Roy, el cual se refiere al nivel de adaptación de un ser humano, entendido como un punto que cambia constantemente y está compuesto por estímulos focales, contextuales y residuales que representan el estándar de la gama de estímulos con el que la persona puede reaccionar por medio de respuestas normales de adaptación. Por ello, la adaptación es un proceso de interacción en la cual se expresan sentimientos y prácticas, condicionadas por creencias, valores, principios y vivencias las cuales pueden ser positivas o no, para los sujetos $(6,7)$.

Los mecanismos innatos son aquellos que están o 
son parte de la psique o genética de cada persona. Estos permiten, que, ante los estímulos, las personas construyan una forma de visión de vida y respondan a ellos. Esto es concordante con lo establecido dentro de la teoría estructural genetista, puesto que los sujetos incorporan tanto capital económico como cultural; entendido este último como los significados significantes ante cualquier situación u objeto.

Estudios relacionados revelan que la experiencia del proceso de morir del paciente, visto desde la perspectiva del profesional de Enfermería, es un acontecimiento que puede afectar su personalidad, ante lo cual puede tomar una postura de rechazo y negatividad o bien, impulsar al profesional en su tarea diaria del cuidado al usuario en estado crítico y en especial al moribundo; pero éste proceso se puede condicionar por distintas situaciones, entre ellas: las experiencias pasadas y propias con la muerte, el nivel de interacción de la enfermera con el usuario y la propia construcción del significado de la muerte de cada profesional $(8-18)$

Diversos estudios se han enfocado en aspectos cuantitativos del proceso y afrontamiento a la muerte y poco se relacionan a profundidad del impacto que les producen estas vivencias. Impactos que suelen conducir a estrés, ansiedad, inasistencias o detrimento de la calidad de la atención. Si bien cuantitativamente es importante la situación, el número no da cuenta de las propias vivencias del personal, por tanto, en este estudio se visualizan las experiencias de la muerte del paciente desde el punto de vista de enfermería, y por ello se plantea dar respuesta a la pregunta ¿Cuáles son las experiencias del personal de enfermería ante el proceso de morir y la muerte de pacientes en un área cuidados críticos en una de un hospital de segundo nivel?

\section{METODOLOGÍA}

El estudio fue de carácter cualitativo, descriptivo, debido a que la experiencia de vivir el proceso de muerte implica saberes subjetivos de los participantes, fue transversal puesto a que se realizaron entrevistas en un momento determinado (19).

La selección de los participantes se llevó a cabo de manera intencional, fueron enfermeras que laboran - laboraron en las unidades de cuidados intensivos de una institución de segundo nivel. Para seleccionarlos se construyó una clasificación tipológica bajo las siguientes características: tiempo de relación con el paciente (igual o mayor a una semana), edad del paciente, experiencias previas ante procesos de muerte y edad del participante. Para la característica de tiempo de relación con el paciente se consideraron dos grupos: 1) una semana de relación y 2) más de una semana de relación con el usuario. Por experiencia ante procesos de muerte se consideró: 1) si habían vivido un proceso o 2) no han vivido el proceso. En cuanto a la edad del paciente se dividió en: 1) menor de edad y 2) mayor de edad, y por último en cuanto a la edad de las enfermeras se consideró dos grupos etarios: 1) menor o igual de 30 años y 2) mayor de 30 años. Por tanto, se construyeron cuatro grupos tipológicos de quienes se buscó seleccionar al menos una participante que cumpliera con los criterios en cada grupo.

El instrumento de recolección de información se constituyó por dos elementos: a) cédula de datos personales y b) guion de entrevista semiestructurada. La cédula de datos sociodemográficos incluyó la edad, sexo, antigüedad laboral, nivel académico, capacitación en tanatología y prácticas religiosas de la enfermera.

El guion de entrevista semiestructurada se planteó en base a los estímulos focales y contextuales, de la enfermera ante el paciente en proceso de morir.

Para el procedimiento de recolección de datos en primer término se sometió a evaluación el protocolo de investigación al Comité de Ética e Investigación del Departamento de Enfermería de la Universidad de Sonora y una vez con el aval se procedió a ubicar a las participantes del estudio -en base a los criterios tipológicos- de forma intencionada, esto es a partir del contacto directo, para posteriormente solicitarles su participación; una vez aceptado, se les entrevistó en las áreas clínicas y durante los espacios en los que tuvieran la oportunidad de ser entrevistadas. A las participantes se les solicitó su consentimiento verbal para audio-grabar las entrevistas.

En cuanto al proceso de análisis de datos sociodemográficos se utilizó estadística descriptiva de tendencia central y dispersión. La información cualitativa recolectada fue audio-grabada en grabadora de voz digital marca Olympus modelo VN-6200PC para posteriormente ser transcrita en su totalidad en el programa F4 y capturar la entrevista al programa NVivo versión 7. Los datos se analizaron mediante procesos inductivos con análisis de las narrativas de las entrevistadas.

La presente investigación se apegó a lo establecido en el Reglamento de la Ley General de Salud en Materia de Investigación, que de acuerdo con el artículo 17 se consideró como sin riesgo ya que se emplearon técnicas y métodos de investigación documental mediante entrevista.

Para proteger la identidad de las participantes se cambió su nombre real por un código, no obstante, se comentó la posibilidad de que aparecieran sus nombres, ante lo cual no hubo inconveniente por parte de las entrevistadas.

\section{RESULTADOS Y DISCUSIÓN}

\section{Características socio demográficas de las participantes}

El estudio se llevó a cabo con siete enfermeras, quienes laboran en una institución de segundo nivel de Hermosillo Sonora. Las edades oscilaron entre los 26 y 50 años, con una media de edad de 34.4 años. Tres de ellas cuentan con 
grado académico de licenciatura, dos con maestría y dos son especialistas.

\section{Mecanismos de Adaptación Innatos}

Uno de los principales mecanismos que suele adoptar el personal de enfermería, y en especial en las primeras experiencias de muerte del paciente, se refiere al mecanismo de justificar la muerte del paciente en función de la edad de este. En diversas sociedades la muerte suele poseer significados diferentes y especialmente en sociedades occidentales donde el adulto mayor ha perdido valor para la funcionalidad del sistema social y por ende los imaginarios hacia el cuidado suelen cambiar, al pasar de considerar que el adulto poseía un alto valor social por su experiencia para el funcionamiento de la comunidad a considerarlo como una carga física, emocional y económica. En contraste, al niño se le asigna un alto valor social, indicador de ellos el tiempo y recursos que se le destinan para conformarlo como un elemento productivo, que permita la reproducción social $(20,21)$. Todo lo anterior derivado de la idea de sociedades modernas, donde prevalece la producción, la satisfacción inmediata, y principalmente la exclusión.

Aspecto que se puede observar en el caso de la entrevistada E1, quién utilizó este mecanismo para disminuir su pesar ante la muerte en función de la edad del paciente.

y si sentí me acuerdo que parada, así como impactada primeramente porque era la primera vez que se me moría un paciente, igual y si, $\mathrm{mmm}$ no quiero ser gacha diciendo no me dio tanta lástima, pero, o sea, ya era una señora mayor, se comprende pues ya por la edad ya [...] (ET).

Por otra parte, y como una estrategia secundaria la interacción con el usuario puede generar un mecanismo de proyección acerca del paciente, y procurar mejorar la calidad de su cuidado, tal como lo expresa E2, quien manifiesto que si bien inicialmente se sintió "parada, impactada", así mismo, y construye un mecanismo de que replantea un sentimiento empático que favorece la relación enfermera paciente ${ }^{(7)}$.

[...] "siempre uno piensa que puede ser su hermano, su tío. No todas tenemos ese pensar, yo sé, cada enfermera es, tiene su forma de atención de ver, pero en mi sí, yo en lo personal a veces pienso que puede ser un familiar tuyo y tratas de darle confort" (E2).

Roy establece que los mecanismos de afrontamiento innatos se tienen como parte de la genética humana, o expresado en otro contexto semántico como humanismo. Esto lo podemos observar en la respuesta de E2, quien refiere que durante el proceso de interacción con el usuario proyecta la situación hacia el cuidado de un familiar; lo anterior defirió con Gálvez, ya que, en lugar de mostrar distanciamiento como una estrategia primaria de defensa, mostró mayor empatía con el usuario ${ }^{(7,24)}$.

\section{Mecanismos de adaptación adquiridos}

Concordante con Roy, las instituciones educativas y de salud establecen diferentes estrategias que ayudan a sus miembros a facilitar su adaptación ante el proceso de muerte. Derivado de ellas, se encuentran los mecanismos de adaptación adquiridos por medio de cursos, diplomados y/o materias que ayuden al profesional (24).

"Y el curso que tome de cuidados paliativos y tanatología me ha ayudado mucho, si te ayuda, yo me pongo y veo el autor que por citar un ejemplo esta está la autora, la Ros, como ella también sufrió y nada todos sus libros, toda su vida todo lo que ella de alguna manera, porque también ella lo paso y lo fue transcribiendo, entonces dices tú bueno, porque no, porque puedo uno no ver la muerte como parte del ciclo de la vida pues" (E3).

El apoyo social a través de grupos y cursos que preparan a los profesionales ante el proceso de morir y la muerte de un usuario han sido sin duda una pieza clave para el proceso de afrontamiento del profesional de enfermería, tal como lo expresó E4, una de las enfermeras entrevistadas.

\section{Estrategias de afrontamiento primarias}

\section{Barreras}

Entre las principales estrategias que desarrollan las enfermeras para afrontar el proceso de muerte del usuario se encuentra el distanciamiento. Los procesos de formación profesional se encuentran inscritos en el concepto de Campo y Habitus desarrollado por Bourdieu (22); en estos se establece que las transmisiones culturales de las formas de trabajo son incorporadas por las enfermeras y operacionalizadas en la práctica, que en el caso del afrontamiento al sufrimiento los formadores refuerzan la idea del distanciamiento emocional hacia el paciente. Si bien, enfermería expresa como una buena práctica el lograr establecer una relación estrecha con los usuarios en la cotidianidad de la práctica, en el caso de pacientes moribundos se observa alejamiento que permite no involucrarse sentimentalmente y por ende evitar el sufrimiento derivado de la muerte del paciente.

"Siempre nos leen la cartilla desde la escuela que no te debes de involucrar, ni sentimental, ni amoroso, ni nada de nada con el paciente, es lo profesional" (E4).

La situación anterior se contrasta en los comentarios expresados por E5 para quien las interacciones con el paciente generan situaciones de acercamiento, las cuales dan lugar a lazos sentimentales, entendidos estos como un 
valor especifico dado por el profesional de enfermería al usuario, los cuales aumentan durante el cuidado y causan con ello un dolor personal al darse el proceso de morir o la muerte.

"amm, pues yo creo que ahí es cuando me bloque, para no sentir, es como que tu mente se bloquea y es de que, no, no tienes que sentir, para no sentir pues, ó sea no se es, no sé cómo explicarlo es para no dejar que te afecte, o sea como no conocía al paciente ni nada dije: no me tiene que afectar, ponemos una barrera para no dejar que nos afecte $y$ vaya más allá pues" (E5).

Roy expresa que el afrontamiento, se consigue por medio de un proceso de interacción en el cual se ven inmiscuidos sentimientos y prácticas, que están condicionadas por creencias, valores, principios y vivencias. Se encontró que las enfermeras entrevistadas durante el proceso de afrontamiento presentan sentimientos de negación mismos que llevan a ejercer una práctica de alejamiento que se ve condicionada con principios aprendidos durante la práctica profesional.

\section{Aceptación}

Otra de las estrategias propuestas por Gálvez(7) dentro del afrontamiento es la aceptación, que desde el punto de vista de Kübler Ross es la aceptación la última etapa del ciclo de duelo de una persona.

"Me sentí triste en el momento, pero igual no deje que se me notará mucho, pero ya meditando y todo el diagnóstico de la paciente pues creo que fue lo mejor que le pudo haber pasado a la bebe" (E5).

A la aceptación se llega después de transitar por cuatro etapas previas (aunque no necesariamente secuenciales) de negación- distanciamiento, irá, negociación y depresión, es decir tras un proceso de meditación o justificación de la muerte del individuo, tal como lo expresó la E5.

\section{Estrategias secundarias}

Como estrategia secundaria el profesional de enfermería tiende a buscar apoyo social en grupos de tanatología, ayuda psicológica o grupos religiosos, que hagan llevadera la labor diaria de lidiar con la muerte y el proceso de muerte de los usuarios.

"Cuando llevamos esta materia yo sentí como que hubo un poquito de cambio porque hicimos muchas actividades con ella con la docente que nos impartió esta materia que nos ayudó mucho para que nosotros nos preparáramos en el sentido que dé hay que dejar ir cuando estamos en estos procesos" (E6).
El apoyo social a través de grupos y cursos que preparan a los profesionales ante el proceso de morir y la muerte de un usuario han sido sin duda una pieza clave para el proceso de afrontamiento del profesional de enfermería.

La importancia de incluir material de apoyo que prepare a los futuros profesionales de la salud a enfrentarse a los procesos de muerte de los usuarios es de suma importancia, ya que éstos salen con armas sustentables para enfrentarse a dicha situación, que si bien es cierto no siempre son suficientes, pero sí de gran ayuda como lo manifiesta la participante E6, quien recibió una materia de tanatología dentro de su formación profesional. Según Blumer en su teoría del interaccionismo simbólico explica que el ser humano orienta sus actos hacia las cosas en función de lo que éstas importan para él (23).

[...] "Fijate que no tiene uno este decir, voy a tratar, tengo este duelo, voy a tratar de un apoyo de un profesionista o de algo para liberar y poder estar tranquila, no, no nunca, te dejas llevar por otra vez, la, la, la, tu rutina y realmente no, no buscas esas ayudas pues, pero... ¿Cómo lo superas? Si, si hubo momentos en el que yo fui a la iglesia o lo que tú quieras y le pedía a Dios por el o sea y que estuviera bien"(E7)

Dentro de las estrategias secundarias de afrontamiento propuestas por Gálvez ${ }^{(7)}$ se encuentra la búsqueda de soporte ya sea, emocional o espiritual, en este caso E7 refiere que el seguir con la rutina laboral y el ir a visitar al usuario fallecido a la iglesia y orar por él ha sido un aliciente durante el proceso de afrontamiento.

\section{CONCLUSIONES}

La muerte y el proceso de morir es sin duda una de las experiencias que marcan la labor diaria del profesional de enfermería, misma que se ve condicionada por experiencias pasadas y actuales en la vida del enfermero (a).

El proceso de morir se condiciona por tres tipos de estímulos contextuales importantes: edad del usuario, la relación enfermera paciente y el tiempo de ésta, el cual se ve definida por la estadía del usuario en el área hospitalaria. Se encontraron procesos de afrontamiento eficaces en aquellas enfermeras que dentro de su preparación profesional han incluido materias, diplomados o cursos en donde se les capacita con herramientas para afrontar el proceso de morir de los usuarios, así como su muerte.

Las principales estrategias de afrontamiento encontrados fueron: barrera, redefinición acerca de la muerte, entendida como una justificación mediada por las ideas sociales de las diferentes etapas de vida, además de soporte emocional por algún tipo de creencia religiosa.

Si bien por la alta cantidad de personal de sexo femenino en enfermería fue más fácil tener contacto con sus experiencias, también se considera es necesario conocer cómo es la experiencia de la muerte en el profesional de 
enfermería masculino, ya que dentro de esta investigación sólo se pudo contar con la colaboración de profesional femenino.

El estudio proporcionó elementos que visualizan experiencias de personal de enfermería que afronta la muerte de pacientes, a partir de ello se propone que se incluya de manera obligatoria, dentro de todos los planes de estudio a nivel licenciatura y posgrado, una materia de tanatología o cuidados paliativos, a su vez se incluya dentro de las unidades hospitalarias un curso- taller de tanatología para personal que labora en unidades de cuidado crítico. Como limitación de estudio se considera que las entrevistas fueron realizadas a mujeres y no se incluyó el discurso de hombres, además se deben considerar en próximas investigaciones la inclusión de diferentes escenarios de cuidados intensivos.

\section{REFERENCIAS BIBLIOGRÁFICAS}

1. García V, Rivas E. Experiencia de enfermeras intensivistas pediátricas en la muerte de un niño: vivencias, duelo, aspectos bioéticos. Cienc. enferm. 2013; 19(2): 111-24.

2. Instituto Nacional de Estadística y Geografía (INEGI). Defunciones generales registradas por entidad federativa. INEGI [Internet]. 2010. [Acceso 02 mayo 2017]. Disponible en: http://www.beta.inegi.org.mx/app/tabulados/pxweb/ inicio.html?rxid=75ada3fe-1e52-41b3-bf27-4cda26e957a7\& $\mathrm{db}=$ Mortalidad\&px=Mortalidad_1

3. Soares F, Vieira M, De Sena R. Significado de la muerte y del morir para los alumnos de enfermería. Rev. Invest. educ. enferm. 2011; 29(3), 407-18.

4. Beraldo $M$, Almeida De, Bocchi $M$. Da frustração ao enfrentamento do cuidado para a morte por técnicos de enfermagem. Rev. Bras. Enferm. 2015; 68(6): 1013-19.

5. Benbunan-Bentata B, Cruz-Quintana F, Roa-Venegas J, Villaverde-Gutiérrez C, Benbunan-Bentata R. Afrontamiento del dolor y la muerte en estudiantes de enfermería: una propuesta de intervención. Rev. International Journal of Clinical and Health Psychology. [Internet]. 2007. [Acceso 22 Agosto 2016] 1 (7). Disponible en: https://dialnet.unirioja.es/ servlet/articulo?codigo $=2251799$

6. Raile Alligood M. Modelos y teorías en enfermería. 8va Ed. Barcelona (Es). Elsevier España. 2015. 728 p.

7. Gálvez-González, M., del Águila-Hidalgo, B., FernándezVargas. L., Muñumel-Alameda, G., Fernádez-Luque, C. y Rios-Gallego, F. Estrategias de afrontamiento ante la muerte del paciente crítico: percepciones y experiencias de enfermeras. Rev. Nure Investigación. [Internet]. 2013. [Acceso 30 de Agosto de 2016]. Disponible en: http://www. nureinvestigacion.es/OJS/index.php/nure/article/view/623
8. Vega-Vega P, González-Rodríguez R, Palma-Torres C, Ahumada-Jarufe E, Mandiola-Bonilla J, Rivera-Martínez S. Develando el significado del proceso de duelo en enfermeras(os) pediátricas(os) que se enfrentan a la muerte de un paciente a causa del cáncer. Rev. Aquichan. [Internet]. 2013. [Acceso 12 abril 2016]; 13 (1). Disponible en: http://www. redalyc.org/articulo.oa?id=74128687009

9. Pérez Vega M, Cibanal L. Impacto psicosocial en enfermeras que brindan cuidados en fase terminal. Rev. Cuidarte [Internet]. 2016. [Acceso 9 mayo 2016]; 7(1). Disponible en: http://dx.doi.org/10.15649/cuidarte.v7i1.295

10. Freitas Tiago L, Banazeski A. Eisele A, De Souza E, Bitencourt J, De Oliveira V, Souza S. The look of Nursing on Death and Dying Process of critically ill patients: an Integrative review. Rev. Enfermería Global. [Internet]. 2016. [Acceso 08 septiembre 2016]; 15 (41). Disponible en: http:// scielo.isciii.es/pdf/eg/v15n41/en_revision2.pdf

11. Pons Diez X. La aportación a la psicología social del interaccionismo simbólico: una revisión histórica. Rev. De psicología y psicopedagogía. [Internet]. 2010. [Acceso 28 mayo 2018]; 1 (9). Disponible en: https://dialnet.unirioja.es/ servlet/articulo?codigo $=3268858$

12. Guerra-Manzo E. Las teorías sociológicas de Pierre Bourdieu y Norbert Elias: los conceptos de campo social y habitus. Rev. JSTOR [Internet]. 2010. [Acceso 02 mayo 2017]; 83 (28). Disponible en: http://www.jstor.org/stable/20749176

13. Castanedo I, Suárez C, Vivar C. Capacitación y afrontamiento de las enfermeras de urgencias ante la muerte y el duelo. Rev. Enfermería Docente. [Internet]. 2010. [Acceso 13 mayo 2016]. Disponible en: http://www.index-f. com/edocente/91pdf/91-016.pdf

14. Lasa A, Vallejo M, Domínguez J. Género y respuesta emocional inducida mediante imaginación. Rev. Psicothema. [Internet]. 2007. [Acceso 11 mayo 2016] 2 (19). Disponible en: http://www.psicothema.com/psicothema.asp?id=3355

15. Bernardes C, Vargas J, Garcia A, da Luz K, de Oliveira Vargas M. Percepção de enfermeira(o)s frente ao paciente oncológico em fase terminal. Rev, Baiana De Enfermagem [Internet]. 2014. [Acceso 15 mayo 2016]; 1 (28). Disponible en: http://dx.doi.org/10.18471/rbe.v28i1.8883

16. Souza e. Souza L, Mota Ribeiro J, Barbosa Rosa R, Ribeiro Gonçalves R, Oliveira e. Silva C, Barbosa D. A morte e o processo de morrer: sentimentos manifestados por enfermeiros. Rev. Enfermería Global. [Internet]. 2013 [Acceso 13 mayo 2016]; 4 (12). Disponible en: http://scielo.isciii.es/ pdf/eg/v12n32/pt_administracion4.pdf 
17. Aguilar Cordero M, Mur Villar N, Padilla López C, García Espinosa Y García Aguilar R. Actitud de enfermería ante el dolor infantil y su relación con la formación continua. Rev. Nutrición Hospitalaria. [Internet] 2012. [Acceso 20 mayo 18]. 6 (27). Disponible en: http://dx.doi.org/10.3305/ nh.2012.27.6.6029

18. DeGrandpre, R. A science of meaning: Can behaviorism bring meaning to psychological science? Rev. American Psychologist. [Internet]. 2000. [Acceso 02 mayo 2018] 7 (55). Disponible en: https://www.ncbi.nlm.nih.gov/ pubmed/10916862

19. Hernández R, Fernández C, Baptista P. Metodología de la investigación. 5ta Ed. México. Mc Graw Hill. 2010. 607 p.

20. Elias Norbert. La soledad de los moribundos. 1987. México: Fondo de Cultura Económica. p. 111.

21. Parsons Talcott. El sistema social. 1999. Primera edición. México: Alianza Editorial. Pag. 534.

22. Bourdieu Pierre. Capital cultural, escuela y espacio social. 2da Ed. México. Editorial Siglo XXI. 2011.184 p.

23.Blumer Herbert. Symbolic Interaction Perspective and Method. 1ra Ed. Englewood Cliffs (NJ). Prentice Hall. 1969. 208 p.

24. Blue C, Brubaker K, Fine J, Kirsch M, Papazian K, Riester M. Sor Callista Roy. Almena A, Huber M. Modelos y teorías en enfermería. $1^{\text {a }}$ Ed. Madrid (Es). Harcourt Brace. 1997. 740 p.

25. Cetma perdigan A, Strosser G. El proceso de muerte y la enfermería: un enfoque relacional, reflexiones teóricas en torno a la atención frente a la muerte. Rev. De Saúde Caletiva. [Internet]. 2015. [Acceso 20 Mayo 2017]. Disponible en: http://www.scielo.br/scielo.php?pid=\$0103$73312015000200485 \&$ script=sci_abstract\&tlng=es 